(5) After washing in chilled Coulfield solution, fix in it for an hour under a vacuous conditions.

(6) Dehydrate and embed in methacrylate.

(7) Cut ultrathin sections.

Crystals of formazan were seen not only on the outer mitochondrial membrane but, also on the cytoplasmic structures, which ware presumed to be endoplasmic reticulum.

The troublesome problem of these procedures was a false positive reaction due to the translocation of formazan, which had been melted into methacrylate.

\title{
Discussion
}

Dr. Otori :

1) The present light microscopic specimens stained with TNBT seem to show diffusion of formazan. Otherwise, is it characteristic of TNBT ?

2) NBT-formazan, assumed to be hardly soluble in organic solvents, is considerably extracted in vitro through organic solvents.

3) Formazan granules in the present electron microscopic studies seem to be excessively few and small.

Dr. Ogawa :

1) Formazans on thick secktions might look diffusive and it is not chacteristic of TNBT.

2) I agree with you concrnieng the solubility of NBT-formazan.

Dr. Shiga: It is desirable to choose stable and hardly water soluble enzymes for histochernical application. And, in dehydration procedure, it is preferable to devise a method not to release formazan from tissue protein. In our experiment, formazan easily liberates through substitution of bound water by alcohol.

Dr. Sugimoto: Polyvinyl alcohol may be used as water soluble embedding medium.

Dr. Okawa : It is reasonable that histochemists are interested in electron microscopic application. However, it must be re-emphasized that methods for electron microscopic histochemistry are to a certain extent different from usual light microscopic histchemistry. Therefore, it is necessary to get conclusions from its findings with great caution.

Dr. Takeuchi : I think that the comparison of electron microscopic data with those of light microscopic observation may be also useful.

\section{Histochemical Distribution of Phosphorylase and Succinic Dehydrogenase Activity in Some Muscle Fibers}

\author{
Masanori Uono, M. D., Takao Muro, M. D., Hitoshi Tanabe, M. D. \\ Satoshi UedA, M. D. and Masanori Tomonaga, M. D. \\ The Third Department of Internal Medicine, (Director: Prof. S. Okinaka, M.D.) \\ Faculty of Medicine, University of Tokyo, Tokyo.
}

Skeletal Muscle fibers have already been classified morphologically and physiologically into two types, namely the white muscle fiber with kinetic and phasic character and the red muscle fiber with tonic and postural character. 
It has also been known that white muscle fibers revealed the high activity of phosphorylase $(\mathrm{PhR})$ and red muscle fibers contained high activity of succinic dehydrogenase $(\mathrm{SD})^{1,2,3,4,5)}$.

Concerning sooth muscle fibers, on the other hand, such types as white and red muscle fibers of the skeletal muscle have not been discriminated. However, it has recently been considered that they showed some specificity in the resting potential, which was appreciated as a fairly notable indicator of the character of smooth muscles. Axelsson, Bueding and Bülbring have investigated the inhibitory effect to the movement of the intestinal smooth muscle and found that adrenaline increased PhR activity in the smooth muscle in accordance with the hyperpolarization, and it was assumed that the activation of $\mathrm{PhR}$ by adrenaline was indirectly brought about by an increase in the formation of cyclic adenosine $-3^{\prime}, 5^{\prime}$-phorsphoric acid $\left(3^{\prime}, 5^{\prime}-A M P\right)$. Such findings suggest that there are some relation between $\mathrm{PhR}$ and the movement of the smooth muscle ${ }^{6,7)}$.

Thus, we attended the following research for the histochemical distribution of $\mathrm{PhR}$ and $\mathrm{SD}$ activity in the smooth muscles of some organs, comparing with the pattern in the skeletal muscle.

\section{Materials and Methods}

The experiments were carried out with adult dogs. The esophagus, the stomach, intestines, the gall bladder, the urinary bladder, the uterus, the scrotum, some arteries and also the heart were chiefly investigated.

$\mathrm{PhR}$ activity was demonstrated after Takeuchi and Kuriaki's iodine method ${ }^{8,9)}$, and SD after Nachlas et al's nitro-BT method ${ }^{103}$.

In order to show an enough contrast of $\mathrm{PhR}$ activity among the organs each other, the time of incubation was shortened (less than 30 minutes) equally in any series.

\section{Results}

In the esophagus $\mathrm{PhR}$ activity was intensely demonstrated both in internal circular and external longitudinal muscle layers, from the lower pharynx to the upper cardia. Any difference between the striated muscle and the smooth muscle was not found (Fig. 1). SD activity was also diffusely shown as PhR in those portions (Fig. 2).

In the cardia, however, some muscle fibers revealed no activity of PhR (Fig. 3). This tendency became more remarkable below the gastric corpus, and in almost all muscle layers, as far as the intestines, $\mathrm{PhR}$ activity was hardly demonstrated in the condition of short incubation (Fig. 4). While SD activity was found moderately throughout the smooth muscle of the intestinal canal, though the activity was somewhat decreased in the portion below the gastric corpus.

The gall bladder revealed some individual difference in $\mathrm{PhR}$ activity, but, when demonstrated, showed high or moderate activity in the smooth 


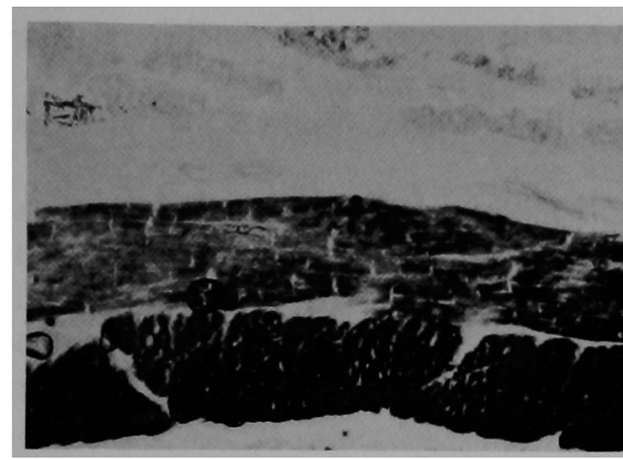

Fig. 1. PhR activity in the esophageal muscle layer $(\operatorname{dog}) .30 \times$

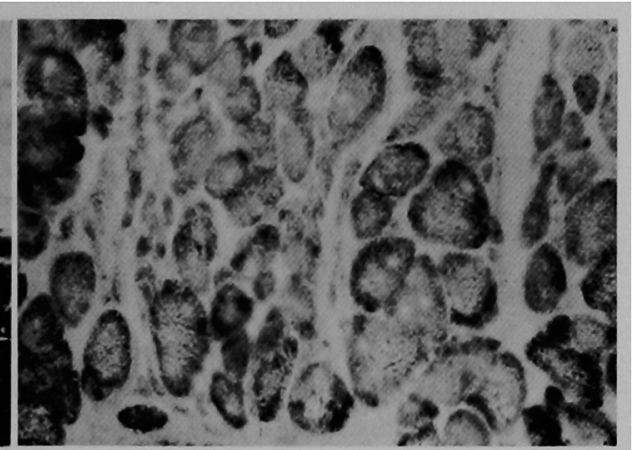

Fig. 2. SD activity in the esophageal muscle layer $(\operatorname{dog}) .100 \times$

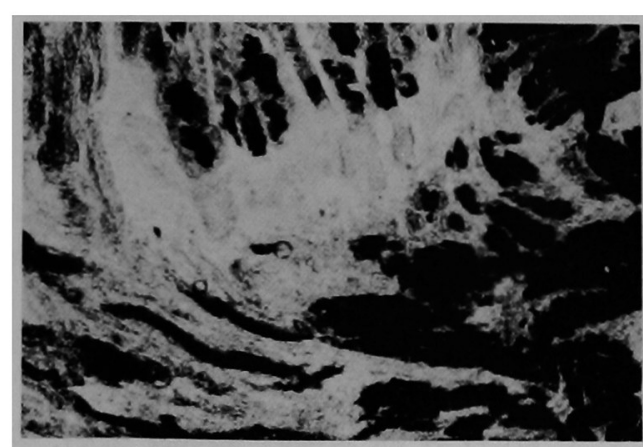

Fig. 3. PhR activity in the muscle layer of the upper part of the cardia $(\mathrm{dog})$. $40 x$

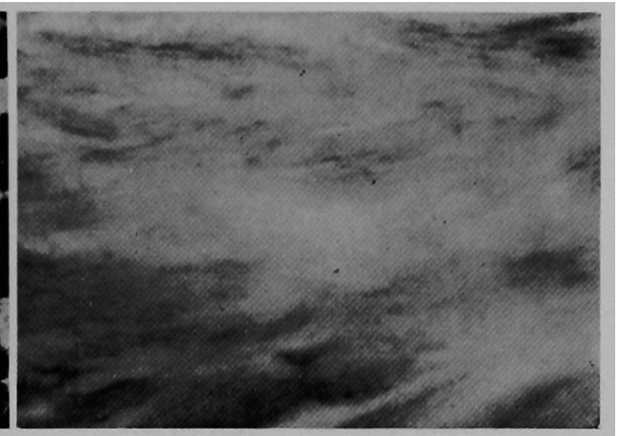

Fig. 4. Poor activity of $\mathrm{PhR}$ in the gastric corpus (dog). 100X

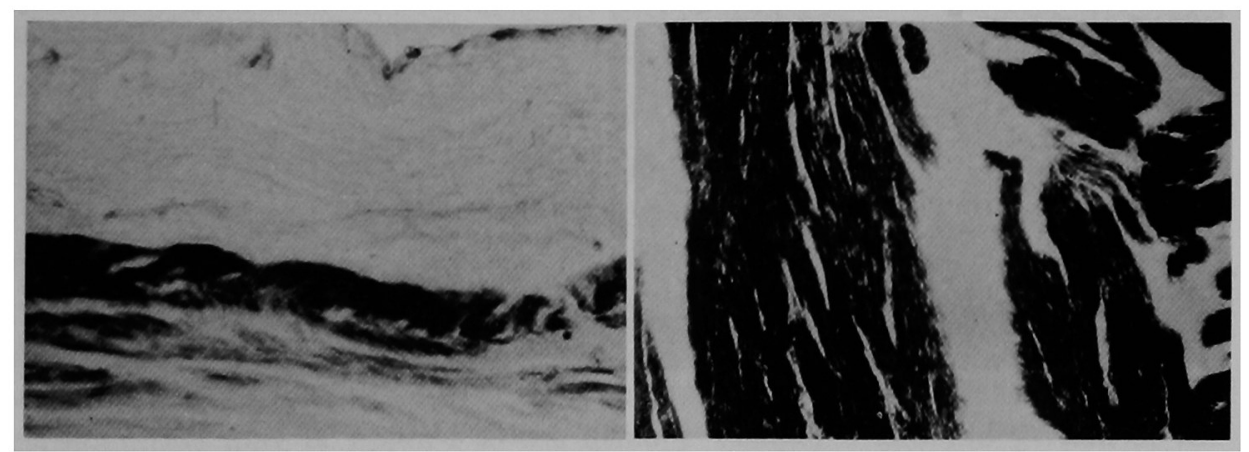

Fig. 5. PhR activity in the muscle layer of the gall bladder (dog). $100 \times$
Fig. 6. PhR activity in the muscle of the urinary bladder (dog). $100 \times$

muscle layer (Fig. 5), while SD activity was poor or almost negative. The difference of these activities between the corpus and the neck of the gall bladder.

In the urinary bladder, $\mathrm{PhR}$ activity was constantly intense, and SD activity was moderate or less in all muscle layers (Fig. 6, 7).

The smooth muscle of the scrotum showed moderate or high activity of $\mathrm{PhR}$ and poor activity of SD.

Concerning the smooth muscle of the uterus, $\mathrm{PhR}$ activity assumed to be 
poor and SD activity to be moderate, but more experimental studies relating the sexual phase were needed, and the details were omitted now.

In the media of the arteries PhR activity was poor, while SD acivity was demonstrated in some degrees (Fig. 8).

In addition, the heart muscle was also investigated. SD activity was much higher than in any other smooth muscles, but the conducting system showed only moderate activity (Fig. 9). PhR activity of the heart muscle exhibited the difference between in the atrium and ventricle, the former demonstrated fairly high and the latter poor activity, and in the conducting system marked activity was observed (Fig. 10).

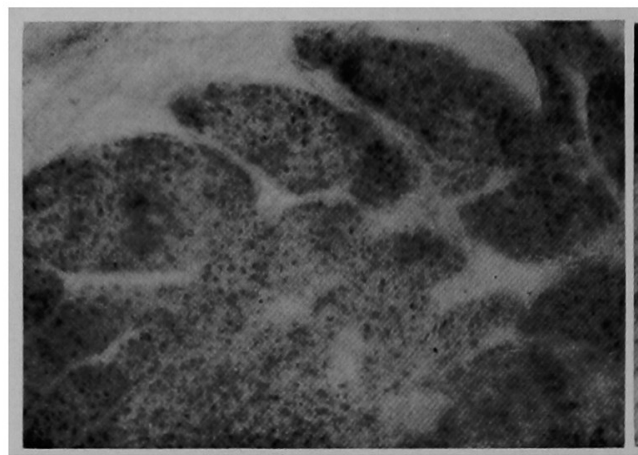

Fig. 7. SD activity in the muscle of the urinary bladder (dog). $100 \times$

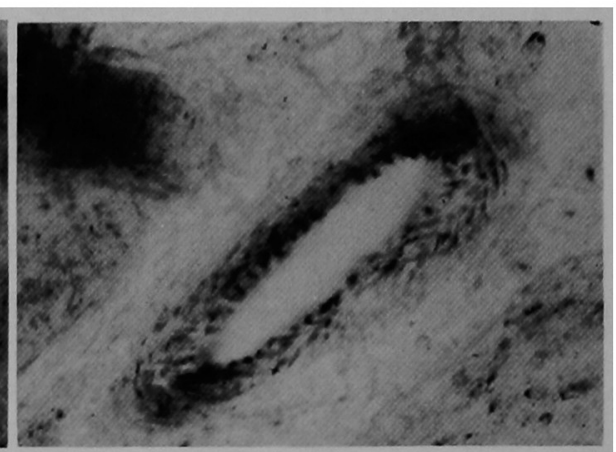

Fig. 8. SD activity in the media of an artery of the urinary bladder $(\operatorname{dog})$. $270 \times$

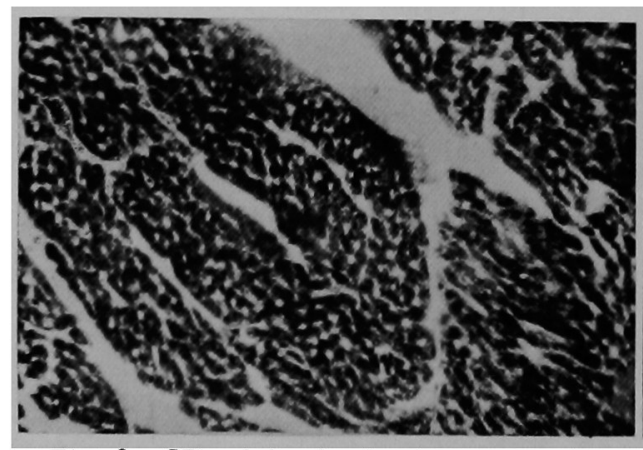

Fig. 9. SD acivity in the heart muscle $(\operatorname{dog}) .100 \times$

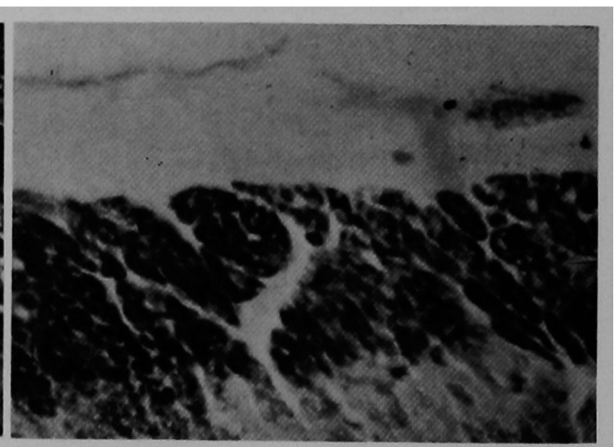

Fig. 10. PhR activity in the heart muscle (dog). $100 \times$

Summarizing these results with some other findings in a table, the characteristics of $\mathrm{PhR}$ and $\mathrm{SD}$ activity among the smooth muscles of some organs were compared (Table 1.).

\section{Discussion}

1. In smooth muscles, though not clearly classified as in skeletal muscles, following characteristics may be estimated at least from the view of the enzyme distribution.

a) In gall bladder, urinary bladder and so on, $\mathrm{PhR}$ was superior to SD, 
Table 1. Characteristics of the distribution of PhR and SD activity.

\begin{tabular}{|c|c|c|c|c|}
\hline Smooth muscle & $\begin{array}{l}\text { Both } \mathrm{PhR} \text { and } \\
\mathrm{SD} \text {, marked } \\
\text { esophagus } \\
\text { cardia (partly) }\end{array}$ & $\begin{array}{l}\qquad \mathrm{PhR}>\mathrm{SD} \\
\text { gall bladder } \\
\text { urinary bladder } \\
\text { scrotum }\end{array}$ & $\begin{array}{l}\qquad \mathrm{PhR}<\mathrm{SD} \\
\text { arteries, } \\
\text { uterus }\end{array}$ & $\begin{array}{l}\text { Both } \mathrm{PhR} \text { and } \\
\mathrm{SD} \text {, poor } \\
\text { intestinal canal } \\
\text { below the } \\
\text { gastric corpus }\end{array}$ \\
\hline Heart muscle & $\begin{array}{l}\text { atrium } \\
\text { (conducting } \\
\text { system*) }\end{array}$ & & ventricle & \\
\hline Skeletal muscle & & & red muscle & \\
\hline Nerve cell & & $\begin{array}{l}\text { sympathetic } \\
\text { ganglion }\end{array}$ & $\begin{array}{l}\text { ggl. ciliare } \\
\text { ggl. Gasseri } \\
\text { Auerbach's and } \\
\text { Meissner's } \\
\text { plexus }\end{array}$ & \\
\hline
\end{tabular}

and the pattern had a resemblance to the white muscle fibers in the skeletal muscle.

b) In heart muscle, especially in the ventricle, SD was superior $\mathrm{PhR}$, and the pattern had a resemblance to the red muscle fibers of the skeletal muscle.

c) In the esophagus and the atrium of the heart, especially in the conducting system, and so on both $\mathrm{PhR}$ and $\mathrm{SD}$ activty was marked, and each character of the white and the red muscle was seemingly combined.

2. In the bundle of the smooth muscle, nothing like in the skeletal muscle, mozaic figure of $\mathrm{PhR}$-positive (SD-negative) and SD-positive ( $\mathrm{PhR}$-negative) fibers was not observed in the same organ, but the difference among each organ was interesting for the study of the metabolism and the function of the smooth muscle.

3. The pattern of the PhR distribution in the digestive canal, rich in the esophagus and poor in below the cardia, was suggestive, some metabolic difference between these portions. We have been demonstrated that Auerbach's plexus of the esophagus containing "nuclear positive type" nerve cells in monoamine oxidase activity, but not observed below the cardia. Such findings were suggestive that the adrenergic innervation related to $\mathrm{PhR}$ activity. Same consideration may be applied to the urinary bladder $^{13)}$ and the atrium of the heart ${ }^{14)}$, in the wall of which small ganglia with nuclear positive type cells in monoanine oxidase activity were frequently found.

4. The striated muscle of the esophagus did not show the difference between the white and the red muscle as in the skeletal muscle concerning the distribution of PhR and SD. Generally, the nerve fibers near the motor end-plate of the skeletal muscle showed no activity of cholinesterase, but in the esophageal striated muscle cholinesterase activity of nerve fibers were demonstrated markedly towards the end-plate ${ }^{12}$. These were likely an example that striated muscle of the esophagus had the intermediate character between the skeletal muscle and the smooth muscle. 


\section{Summary}

The characteristics of the distribution of $\mathrm{PhR}$ and in the smooth muscles of several organs were studied with normal adult dogs. Some discussion were done comparing with the white and the red muscle fibers in the skeletal muscle, with adrenaline metabolism and with our previous results concerning the distribution of monoamine oxidase and cholinesterase activity.

\section{Acknowledgment}

Authors' acknowledgment is due to Prof. Dr. S. Okinaka and Ass. Prof. Dr. M. Yoshikawa for their valuable guidance and advises.

\section{References}

1) Kondo, M. : Okayama J. Med., $71: 3569,1959 . \quad 2)$ Nachims, V. T. et al. : J. Biophys. Biochem. Cyt., 4:47, $1958 . \quad$ 3) Ogata, T.: Acta Med. Okayama, $12: 215,1958 . \quad 4)$ Okajima, K.: Okayama J. Med., $71: 4299,1959 . \quad 5)$ Saito, K. : Okayama J. Med., 71 : 4377, 1959. 6) Axelsson, J. et al. : J. Physiol., $156: 357,1951$. 7) Axelsson, J. et al. : J. Physiol., $156: 344,1955 . \quad$ 8) Takeuchi, T. : J. Histochem. Cytochem., $3: 153,1955$. 9) Takeuchi, J. Histochem. Cytachem., $8: 277$. 1960. 10) Nachlas, M. M. et al. : J. Histochem. Cytochem., $5: 420$, 1957. 11) Tanabe, H. : Jap. J. Med. Prog., $48: 106,1961$. 12) Okinaka, S. et al. : Proc. Jap. Histochem. Ass., $2: 173,1961$. 13) Tanabe, H. et al. : The 11th Congress of Japanese Branch of International Association of Autonomic Nervous System, Tokyo, $1962 . \quad$ 14) Tomonaga, M. : Jap. J. Med. Prog., 49 : 257, 1962.

\section{Discussion}

Dr. Takeuchi : I am pleased you applied our method for the detection of phosphorylase activity. I would like to add some data. The muscles of the stomach and intestine in various animals (including dog) showed marked phosphorylase activity, and the difference of the activity between outer and inner muscle layers were sometimes observed. In addition, muscle cells of the stomach showed the enzyme activity in specific structure of nuclei.

Dr. Tanabe: In the present studies, the weak reaction in the stomach and other tissues might result from limited incubation time ( 30 minutes) to compare the relative activities. These tissues surely stained through the loner incubation. The best differentiation of the activity in the striated muscles were obtained through the present limited incubation, and. in the case of the smooth muscles of the stomach, the strong activity in pars preventricularis clearey differed from the weak reaction in pars glandularis.

Dr. Otsuka: In the heart, succinic dehydrogenase reaction is almost negative in the fibers of the impulse conducting system, in contrast with the strong reaction in other cardiac muscles.

Dr. Tanabe: The present studies emphasized the strong phosphorylase activity in the impulse conducting system. Further studies will compare it with succinic dehydrogenase activity.

Dr. Yasuda: Have the phosphorylase activity a parallel relataionship between adrenalin secretion? In our experiment, phosphorylase activity in the apocrine glands is considerably porallel with cholinesterase activity.

Dr. Tanabe: In the present studies, the tissues that combined with adrenergic transmission showed the marked phosphorylase activity. On the other hand, the reactions in some tissues combined with cholinergic transmission were relatively weak. The adrenergic ganglion in the present studies also showed strong cholinesterase activity. 